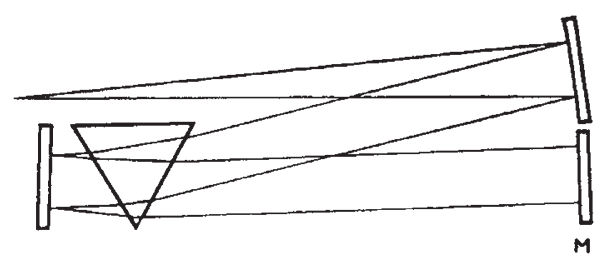

Fig. 1. Littrow system with additional plane mirror $M$, as used by Dr. W. C. Price

spectrometer and has the advantage that the small angle off-axis in a vertical plane. (about $1^{\circ}$. in the $S 3$ spectrometer) tends to reduce the curvature of image already mentioned. In practice, it is unnecessary to make any alteration to the slits, which is a point of some practical importance.

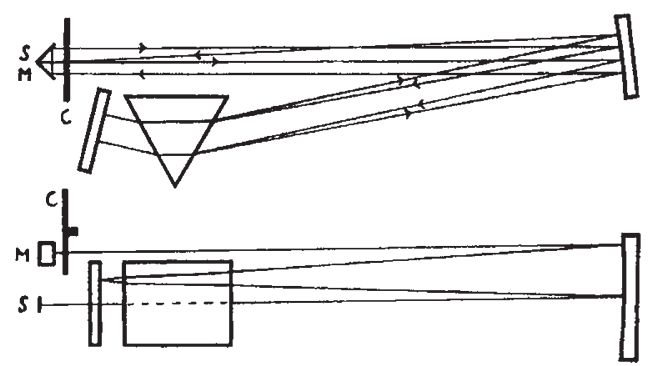

Fig. 2. Plan and elevation illustrating a simple method of converting a single- to a double-monochromator. $S$ is the entrance (and exit) slit and $M$ a pair of plane mirrors mounted at right

The elimination of stray light by chopping after initial dispersion has already been described ${ }^{2}$. In our arrangement the chopper $C$ is placed at the position shown in Fig. 2. If the primary spectrum is imagined spread out horizontally across the pair of mirrors, only a small band of wave-lengths will be intercepted by them. If stray light of some other wave-length happens also to fall on these mirrors, it will, of course, be interrupted, but will be separated from the wanted radiation during the further traversals of the prism.

J. C. O. Rocheister

A. E. MARTIN

Sir Howard Grubb, Parsons and Co.,

Optical Works, Walker Gate,

Newcastle upon Tyne 6. June 8.

${ }^{1}$ Walsh, A., Nature, 187, 810 (1951).

${ }^{2}$ Perkin-Elmer Instrument News, 1, No, 4, 3 (1950).

\section{The Xi-Phase and a New Sigma Phase}

Stx phases with structures isomorphous with the iron-chromium sigma phase have been reported in binary alloys of the transition elements of the first long period ${ }^{1-3}$. In addition, Goldschmidt ${ }^{4}$ has shown that the phase FeMo, stable between $1,180^{\circ}$ and $1,540^{\circ} \mathrm{C}$., has the same structure. In the binary system cobalt - molybdenum, a similar phase $\mathrm{Co}_{2} \mathrm{Mo}_{3}$ exists between $1,250^{\circ}$ and $1,620^{\circ}$ C. ${ }^{5}$; recent work in this Laboratory has shown that $\mathrm{Co}_{2} \mathrm{Mo}_{3}$ has a sigmaphase structure. The interplanar spacings of the alloy containing 61 atomic per cent molybdenum are very similar to those of the alloy with 50 atomic per cent FeMo given by Goldschmidt, except that they are slightly smaller, as might be expected when the slightly smaller cobalt atom replaces iron. Multiplying the $\mathrm{Co}_{2} \mathrm{Mo}_{3} d$-values by 0.981 gives values which agree well with those for $\mathrm{FeCr}$ given by Goldschmidt ${ }^{4}$.

As in the iron - molybdenum system, the cobaltmolybdenum sigma-phase has only a limited temperature-range and decomposes peritectoidally to form the xi-phase $\mathrm{Co}_{7} \mathrm{Mo}_{6}$, which has a rhombohedral structure stable over a wide range of temperature. It is of interest to compare the occurrence of the sigma- and xi-phase in the binary systems of iron and cobalt with chromium, molybdenum and tungsten respectively. Where the second element is in the same period the only intermediate phase is sigma, which has a wide stability range. With molybdenum, both iron and cobalt form a sigma-phase, though this has only a restricted stability-range and decomposes to the xi-phase. In the case of tungsten, the xi-phase only is found. The reason for this transition does not appear to be predominantly a size effect, as there is little difference in the atom sizes of molybdenum and tungsten; it is more probable that it is an electronic one. The xi-phase may thus also be an electron compound, as has been suggested by Sully ${ }^{6}$ for the sigma-phase ; as is the case with the sigmaphase, the proportions of molybdenum and tungsten are greater in the cobalt phases than in the iron ones.

Research Laboratory,

$$
\text { D. SUMMERS-SMTTH }
$$

Associated Electrical Industries, Ltd.,

Aldermaston, Berkshire. June 19.

'Wever, F., and Jellinghaus, W., Mitt. K.W. Inst. Eisenforsch., 13, 93 and 143 (1931).

'Sully, A. H., and Heal, T. S., Researeh, 1, 288 (1948).

a Pearson, W. B., Christian, J. W., and Hume-Rothery, W., Nature, 167, 110 (1951).

- Goldschmidt, H. S., Research, 2, 343 (1949).

${ }^{5}$ Sykes, W. P., and Graff, H. F., Trans. Amer. Soc. Met., 23, 249 (1935).

- Sully, A. G., Nature, 167, 365 (1951).

\section{Separation of Enzymes by Paper Chromatography}

IN a recent publication ${ }^{1}$ from this laboratory, a technique for the study of the chromatographic behaviour of enzymes on paper was described. The ease with which enzymes can be readily identified on the paper chromatogram by the agar-plate method enabled us to study the chromatographic behaviour of some of the important enzymes. In the course of preliminary experiments with amylases, phosphorylases and phosphatases from various sources, we found that some enzymes did not move at all while others moved considerable distances on the paper. This observation prompted us to attempt the separation of enzymes by paper chromatography.

Apart from the work of Mitchell, Gordon and Haskins ${ }^{2}$, who report that some separation of adenosine deaminase from amylase has been achieved by their 'chromatopile' method, no really complete separation of enzymes by paper chromatography has been reported. In a recent note by Wallenfels and Pechman ${ }^{3}$, which appeared after we sent our communication ${ }^{1}$ on the technique for the detection of enzymes on paper, the separation of the enzymes of a mould extract by a micro-electrophoretic technique was described. However, by adopting the usual paper chromatographic technique and using aqueous acetone or sodium chloride or a suitable com- 